\title{
MOLECULAR CLOUD CORES AND PROTOSTARS: OFFSPRINGS OF GRA VITY AND COSMIC MAGNETISM
}

\author{
TELEMACHOS CH. MOUSCHOVIAS \\ University of Illinois at Urbana-Champaign \\ Departments of Physics and Astronomy \\ 1011 West Springfield Avenue
}

Urbana, IL 61801, U. S. A.

\begin{abstract}
The formation of cloud cores (or fragments) and their evolution into protostars are the inevitable outcome of the struggle between gravity and magnetic fields, with ambipolar diffusion as the agent employed to weaken gravity's fierce opponent. The very specific and crucial role of magnetic fields in star formation deduced from detailed quantitative calculations is summarized. Criteria for collapse against magnetic and thermalpressure forces are given. Magnetic braking time scales for both aligned and perpendicular rotators, and ambipolar diffusion time scales in both quasistatically and dynamically contracting cores are presented, and their implications are discussed. The possible role of magnetic fields in the determination of the initial (stellar) mass function (IMF) is beginning to emerge. New calculations on the axisymmetric collapse of clouds due to ambipolar diffusion reveal that the relation $B_{c} \propto \rho_{c}{ }^{1 / 2}$ between the magnetic field strength and the gas density in typical cloud cores holds even in the presence of ambipolar diffusion up to densities $\sim 10^{9} \mathrm{~cm}^{-3}$. Small masses, high densities, and strong fields observed in $\mathrm{H}_{2} \mathrm{O}$ masers are consistent with theoretical calculations.
\end{abstract}

\section{ELEMENTS OF A THEORY OF STAR FORMATION}

Observations of interstellar magnetic fields have been reviewed recently and have been compared with theoretical predictions (Heiles 1987; Mouschovias 1981, 1987a). Remarkable agreement is found between theory and observations from scales of $\sim 1 \mathrm{kpc}$ to scales of $\sim 10^{-3} \mathrm{pc}$. We summarize the key elements of a theory of star formation in magnetic clouds presented recently (Mouschovias 1987a), which accounts for the results of the most recent calculations and observations and which thus represents a refinement of the original scenario proposed more than a decade ago (Mouschovias 1977, 1978). This includes new quantitative results which necessitate significant qualitative changes in traditional ideas concerning star formation. The schematic diagram on the following page summarizes this detailed but still incomplete theory. A dashed line signifies a possible but unlikely evolutionary path. Collapse means indefinite contraction, but not necessarily free fall.

\subsection{Cloud Formation and Early Evolution: Critical Mass, and Magnetic Braking}

The most likely mechanism responsible for the formation of interstellar clouds and cloud complexes is the Parker (1966) instability triggered in the galactic disk by spiral density waves or by any other large-scale disturbance (Mouschovias 1974, 1975a; Mouschovias, Shu, 
A SCENARIO FOR STAR FORMATION IN MAGNETIC CLOUDS

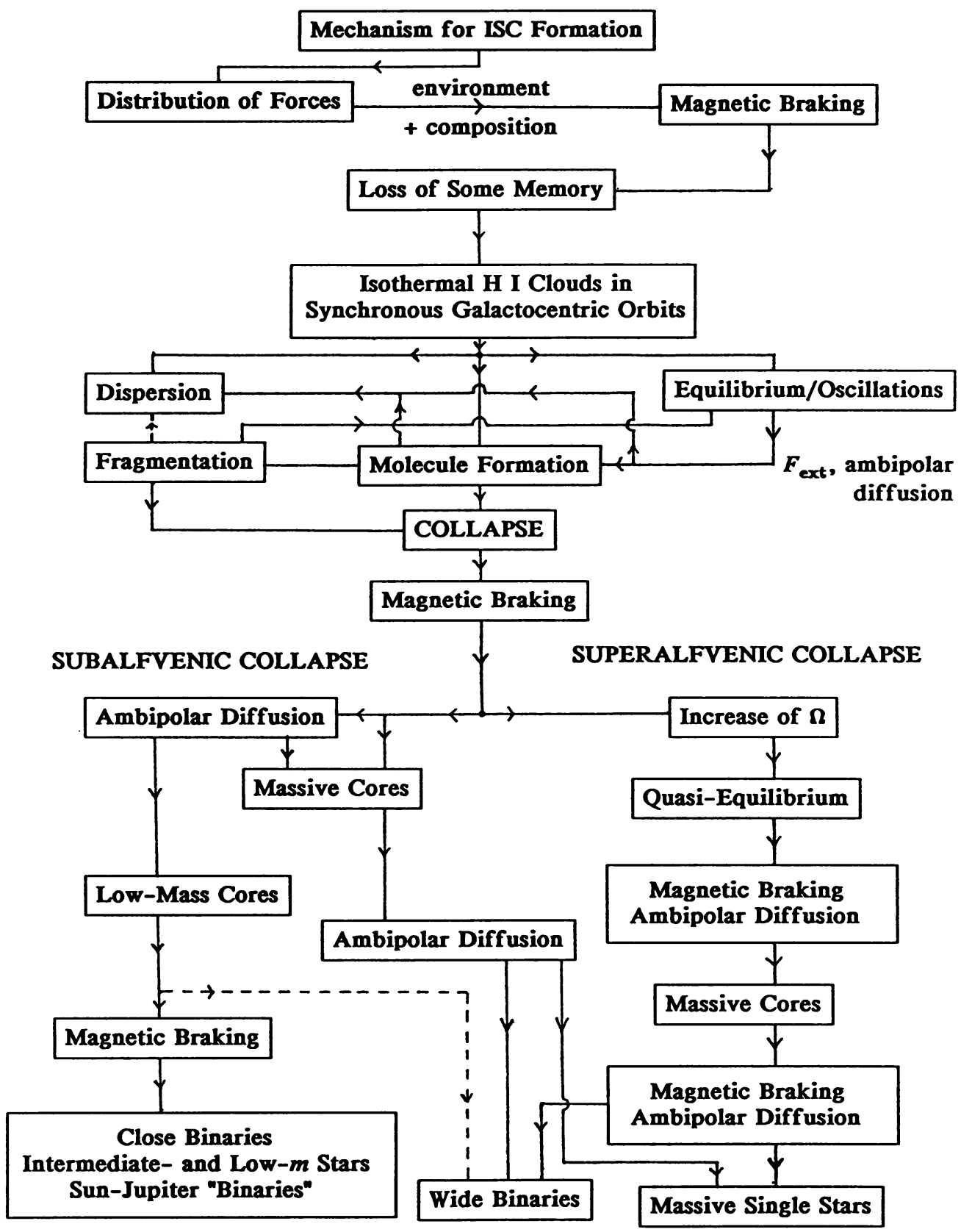


and Woodward 1974; Shu 1974; Blitz and Shu 1980). The thermal instability (Field 1965) and the Jeans instability (Jeans 1928) cannot be responsible for the formation of typical clouds (see detailed discussion in the review by Mouschovias 1981, § 4). Regardless of the nature of this mechanism, its significance stems mainly from the fact that the mechanism determines the distribution of forces (such as gravitational, magnetic, thermal, and centrifugal) within a cloud. Soon after a cloud's formation, thermal-pressure and centrifugal forces lose memory of initial conditions. The differential mass-to-flux ratio $\left.\left[d m\left(\Phi_{\mathrm{B}}\right) / d \Phi_{\mathrm{B}}\right)\right]$ determines the relative magnitude of gravitational and magnetic forces, and affects crucially the subsequent evolution of a cloud (see Mouschovias 1976a,b). One can obtain reasonable theoretical estimates for this function, but no observational determination exists as yet. Observations such as those by Bregman et al. (1983) and by Schwarz et al. (1986) constitute an important first step toward the determination of $d m / d \Phi_{B}$.

For a cloud to collapse, two conditions must be satisfied. First, its mass-to-flux ratio must exceed a critical value given by (see Mouschovias and Spitzer 1976)

$$
\left(\frac{M}{\Phi_{\mathrm{B}}}\right)_{\mathrm{crit}}=\frac{0.53}{3 \pi}\left(\frac{5}{G}\right)^{1 / 2}=\frac{0.126}{G^{1 / 2}} \simeq\left(\frac{1}{63 G}\right)^{1 / 2} .
$$

Second, the external pressure must also exceed a critical value given by $P_{\text {ext }}>P_{\text {crit }}=1.89$ $C^{8} G^{-3} M^{-2}\left[1-\left(M_{\text {crit }} / M\right)^{2}\right]^{-3}$, where $M \geq M_{\text {crit }}, C \equiv\left(k_{\mathrm{B}} T / \mu m_{\mathrm{H}}\right)^{1 / 2}$ is the isothermal speed of sound in the gas, $k_{\mathrm{B}}$ the Boltzmann constant, and $\mu$ the mean mass per particle in units of the atomic hydrogen mass $m_{\mathrm{H}}$. By solving equation (1) for $M_{\text {crit }}$ and substituting in the expression for $\boldsymbol{P}_{\text {crit }}$, one obtains a single sufficient condition for the collapse of selfgravitating clouds supported by magnetic and thermal-pressure forces, namely,

$$
M \geq M_{\mathrm{TM}}=1.38\left\{1-\left[\frac{0.126}{G^{1 / 2}\left(M / \Phi_{\mathrm{B}, \mathrm{cl}}\right)}\right]^{2}\right\}^{-3 / 2} \frac{C^{4}}{\left(G^{3} P_{\mathrm{ext}}\right)^{1 / 2}} .
$$

where $\Phi_{\mathrm{B}, \mathrm{cl}}$ and $M$ are the total flux and mass of the cloud, respectively. Since the total mass-to-flux ratio $M / \Phi_{\mathrm{B}, \mathrm{cl}}$ of initially spherical clouds threaded by a uniform magnetic field is related to the central mass-to-flux ratio $\left(d m / d \Phi_{\mathrm{B}}\right)_{\mathrm{c}}$ by $M / \Phi_{\mathrm{B}, \mathrm{cl}}=(2 / 3)\left(d m / d \Phi_{\mathrm{B}}\right)_{\mathrm{c}}$ (see Mouschovias 1976a, eq. [44]), equation (2a) can equivalently be written in terms of the central mass-to-flux ratio as

$$
M_{\mathrm{TM}} \simeq 1.38\left\{1-\left[\frac{0.19}{G^{1 / 2}\left(d m / d \Phi_{\mathrm{B}}\right)_{\mathrm{c}}}\right]^{2}\right\}^{-3 / 2} \frac{C^{4}}{\left(G^{3} P_{\mathrm{ext}}\right)^{1 / 2}}
$$

Recent numerical determination of critical masses by Tomisaka et al. (1988) is completely consistent with equations (2a) and (2b). The effect of subsonic turbulence is easily included in equation (2a) or (2b), if one desires, as an increase of the isothermal sound speed $C$ and of $\boldsymbol{P}_{\text {ext }}$. However, since magnetic forces dominate thermal-pressure forces at typical molecular cloud densities, one is not usually concerned with the effect of subsonic (and, therefore, subAlf venic) turbulence on the critical mass. An observationally usuful expression is obtained by rewriting the necessary condition (1) for collapse in terms of a critical mean column density of matter, commonly referred to as surface density, as $\left\langle\sigma_{\mathrm{m}}\right\rangle_{\text {crit }}=$ $(1 / 63 G)^{1 / 2}\langle B\rangle=2.4 \times 10^{-2}\langle B\rangle /(50 \mu \mathrm{G}) \mathrm{g} \mathrm{cm}^{-2}$, where $\sigma_{\mathrm{m}} \equiv M / \pi R^{2}$, and $R$ is the cloud radius. Other forms of this equation, for example, in terms of a critical visual extinction, are given in Mouschovias (1987a). This equation implies that clouds, as opposed to cores, with supercritical masses should be very rare. 
A cloud which has not contracted sufficiently to become self-gravitating could either disperse once the cause of its formation is removed or exist in pressure equilibrium with the intercloud medium. In the latter case, it will await an external disturbance (such as a supernova shock wave or an ionization front) to either disperse or implode it --or implode part of it and disperse the rest. Theoretical considerations, as seen above, and the observational fact that velocity fields characteristic of gravitational collapse are not observed suggest that very few, if any, clouds acquire a supercritical mass-to-flux ratio from the outset and begin to collapse as a whole. Instead, clouds reach first a relatively quiescent state (near hydrnstatic equilibrium with magnetic and thermal-pressure forces balancing gravity), with possible leftover stable oscillations about the equilibrium state (Mouschovias 1975b). Centrifugal forces remain small at this stage due to magnetic braking, which operates over a time scale strictly smaller than (see Mouschovias 1977, 1978, 1979a; Mouschovias and Paleologou 1980; or review by Mouschovias 1987b)

$$
\tau_{\|}=\frac{\rho_{\mathrm{cl}}}{\rho_{\mathrm{ext}}} \frac{Z}{v_{\mathrm{A}, \mathrm{ext}}} \equiv \frac{\sigma_{\mathrm{m}, \mathrm{cl}}}{2 \rho_{\mathrm{ext}} v_{\mathrm{A}, \mathrm{ext}}} \equiv\left(\frac{\pi}{\rho_{\mathrm{ext}}}\right)^{1 / 2} \frac{M}{\Phi_{\mathrm{B}}} \equiv 0.4\left(\frac{\rho_{\mathrm{cl}}}{\rho_{\mathrm{ext}}}\right)^{1 / 2} \frac{M}{M_{\mathrm{crit}}} \tau_{\mathrm{ff}},
$$

where $\rho_{\mathrm{cl}}, R$, and $Z$, are the density, equatorial and polar radius, respectively, of a cloud (or fragment) rotating about its $(z-)$ axis of symmetry which is aligned with the magnetic field. The matter density and Alfven speed in the external medium (or envelope) are denoted by $\rho_{\text {ext }}$ and $v_{A, e x t}$, respectively, and the free-fall time $\tau_{\text {ff }}$ is given by equation (6a) below. Since the magnetic braking time scale for a perpendicular rotator has been found to be $\tau_{\perp} \ll \tau_{\|}$ (see Mouschovias and Paleologou 1979; or summary in Mouschovias 1987b, $\S 2.3 .2$ ), most clouds and fragments tend to become aligned rotators in time. For $\rho_{\mathrm{cl}} / \rho_{\mathrm{ext}} \gg 1, \tau_{\perp}$ becomes

$$
\tau_{\perp}=\frac{1}{2}\left(\frac{\rho_{\mathrm{cl}}}{\rho_{\mathrm{ext}}}\right)^{1 / 2} \frac{R}{v_{\mathrm{A}}(R)} \equiv 2\left(\frac{\pi}{\rho_{\mathrm{cl}}}\right)^{1 / 2} \frac{M}{\Phi_{\mathrm{B}}} \equiv(2 / 3)^{1 / 2} \frac{M}{M_{\mathrm{crit}}} \tau_{\mathrm{ff}},
$$

where $v_{\mathrm{A}}(R)=B(R) /\left(4 \pi \rho_{\text {ext }}\right)^{1 / 2}$ is the Alfven speed just outside the cloud surface. It is indeed very clear from equations (3a) and (3b) that, in general, $\tau_{\perp} \ll \tau_{\|}$. The expressions for $\tau_{\|}$and $\tau_{\perp}$ can easily be recovered by simply calculating the time it takes for the torsional Alf vên waves generated by the rotation of a cloud (or core) to affect a moment of inertia in the surrounding medium equal to the moment of inertia of the cloud (or core) (see Mouschovias and Paleologou 1979, 1980; or review by Mouschovias 1987b, in which alternative forms of $\tau_{\perp}$ and $\tau_{\|}$are also given, including the effect of field lines fanning out away from an aligned rotator and, therefore, the result presented here by Nakano).

Molecules form over time scales shorter than evolutionary time scales once the density becomes sufficiently large. Collapse may receive a boost or even be initiated by the decrease in sound speed due to the larger molecular weight and the possible decrease in temperature that may accompany the formation of molecules and/or the decay of subsonic turbulence. The electrical conductivity is so large that the magnetic field remains frozen in the matter (Spitzer 1962) and lengthens the collapse time scale (even after the onset of ambipolar diffusion in the core; see below) by at least one order of magnitude compared to the characteristic free-fall time (Mouschovias 1983; Mouschovias, Paleologou, and Fiedler 1985).

\subsection{Onset, Essence, Time Scales, and Consequences of Ambipolar Diffusion}

Ambipolar diffusion is unavoidable in the core of a self-gravitating, magnetically supported cloud. The essence of ambipolar diffusion is to permit self-gravity to redistribute mass in the central flux tubes of a cloud and thereby induce fragmentation (Mouschovias 1977, 1978, 1979b; see also discussion associated with eq. [8] below), recently being referred to as core 
formation, followed eventually by gravitational collapse of dense cores that have exceeded the critical mass-to-flux ratio specified by equation (1). If ambipolar diffusion in cloud cores can prevent the magnetic field strength from increasing much above a few milligauss up to densities $10^{11-12} \mathrm{~cm}^{-3}$, then the bulk of the (or even the entire) magnetic flux problem of star formation will be resolved. This is so because the ratio of the flux of a protostellar fragment and the flux of a magnetic star (of the same mass) is

$$
\frac{\Phi_{\mathrm{B}, \mathrm{fr}}}{\Phi_{\mathrm{B}, *}}=\frac{\pi B_{\mathrm{fr}} R_{\mathrm{fr}}^{2}}{\pi B_{*} R_{*}^{2}}=\frac{B_{\mathrm{fr}}}{B_{*}}\left(\frac{\rho_{*}}{\rho_{\mathrm{fr}}}\right)^{2 / 3} \simeq\left(\frac{B_{\mathrm{fr}} /\left(10^{-3} \mathrm{G}\right)}{B_{*} /\left(10^{4} \mathrm{G}\right)}\right)\left(\frac{10^{12} \mathrm{~cm}^{-3}}{n_{\mathrm{n}}}\right)^{2 / 3},
$$

where we have taken $\rho_{*} \simeq 0.3 \mathrm{~g} \mathrm{~cm}^{-3}$ as a representative mean density of magnetic A stars. The distinction between the onset of rapid ambipolar diffusion and the actual resolution of the magnetic flux problem is of the essence. Approximate analytical calculations (Mouschovias 1977, 1978, 1979b) as well as one-dimensional collapse calculations (Paleologou and Mouschovias 1983; Mouschovias, Paleologou, and Fiedler 1985) have demonstrated that rapid ambipolar diffusion can indeed set in by the stage $n_{\mathrm{n}} \sim 10^{6} \mathrm{~cm}^{-3}$ in cloud cores, and can have very important effects on the core's evolution. This has recently been confirmed by 2D (cylindrical) and 3D (axisymmetric) collapse calculations by Mouschovias and Morton (1990) and Fiedler and Mouschovias (1990), respectively. However, the issue of whether ambipolar diffusion can resolve the magnetic flux problem is still not completely settled (see below).

Up to the stage that ambipolar diffusion increases the mass-to-flux ratio of the core above the critical value given by equation (1), the contraction remains quasistatic and the time scale $\tau_{\mathrm{AD}}$ for ambipolar diffusion (in an axisymmetric geometry) can be obtained analytically (see Mouschovias 1989, eq. [20]):

$$
\tau_{\mathrm{AD}}=\frac{8}{1.4 \pi^{2}} \frac{\tau_{\mathrm{ff}}^{2}}{\tau_{\mathrm{ni}}}\left(1-8 \tau_{\mathrm{ff}}^{2} / \pi^{2} \tau_{\mathrm{s}}^{2}\right)^{-1}, \quad \text { for } 8 \tau_{\mathrm{ff}}^{2} / \pi^{2} \tau_{\mathrm{s}}^{2}<1,
$$

where $\tau_{\mathrm{ff}}, \tau_{\mathrm{s}}$, and $\tau_{\mathrm{ni}}$ are the spherical free-fall time, the sound crossing time, and the neutral-ion collision time, respectively, and are given by

$$
\tau_{\mathrm{ff}}=(3 \pi / 32 G \rho)^{1 / 2}, \quad \tau_{\mathrm{s}}=r_{\mathrm{c}} / C, \quad \tau_{\mathrm{ni}}=\left(m_{\mathrm{i}}+m_{\mathrm{n}}\right) / \rho_{\mathrm{i}}\langle\sigma w\rangle_{\mathrm{in}} .
$$

All time scales refer to the core, which has radius $r_{c}$. The quantity $\langle\sigma w\rangle_{\text {in }}$ is the average collisional rate between ions of mass $m_{\mathrm{i}}$ (and density $\rho_{\mathrm{i}}$ ) and neutrals of mass $m_{\mathrm{n}}$; it is equal to $1.69 \times 10^{-9} \mathrm{~cm}^{3} \mathrm{~s}^{-1}$ for $\mathrm{HCO}^{+}-\mathrm{H}_{2}$ collisions (see McDaniel and Mason 1973) --note that $m_{\mathrm{i}} / m_{\mathrm{n}}=14.4$ for this particle pair. For magnetically supported clouds the term in brackets in equation (5) is essentially equal to unity and the expression for the ambipolar diffusion time scale in the form $\tau_{\mathrm{AD}} \sim \tau_{\mathrm{ff}}{ }^{2} / \tau_{\mathrm{ni}}$, valid for quasistatic contraction, is a property of the general equations governing ambipolar diffusion and does not depend on geometry (see Mouschovias 1987b, $\S 3.3$ and 3.4). For example, the constant on the right-hand side of equation (5), which refers to an axisymmetric geometry, is $8 / 1.4 \pi^{2}=0.58$, and the constant in cylindrical geometry is $2 / 1.4 \pi=0.45$ (Mouschovias 1979b; or review 1987a, eq. [12a]). In the cores of magnetically supported clouds, equation (5) can be put in the useful form $\tau_{\mathrm{AD}} \simeq$ $2 \times 10^{5}\left(x / 10^{-8}\right) \mathrm{yr}$, where $x \equiv n_{\mathrm{i}} / n_{\mathrm{H2}}$. The characteristic time $\tau_{\Phi}$ for the reduction of the magnetic flux of a core is actually smaller than $\tau_{\mathrm{AD}}$ by exactly a factor of 2 .

After ambipolar diffusion has increased the mass-to-flux ratio of a quasistatically contracting core of a typical molecular cloud above the critical value given by equation (1), accelerated contraction may ensue (if thermal pressure cannot support the core), in which case equation (5) no longer describes the rate at which ambipolar diffusion progresses. We find instead that (see Mouschovias 1989, eq. [23]) 


$$
\begin{aligned}
\tau_{\mathrm{AD}} & =0.27 \frac{\tau_{\mathrm{ff}}^{2}}{\tau_{\mathrm{ni}}}\left(\frac{\Phi_{\mathrm{B}, \text { crit }}}{\Phi_{\mathrm{B}}}\right)^{2}, \quad \text { for } \quad \Phi_{\mathrm{B}} \leq \Phi_{\mathrm{B}, \text { crit }} ; \\
& =1.3 \times 10^{3}\left(\frac{n_{\mathrm{i}} / n_{\mathrm{H}_{2}}}{10^{-10}}\right)\left(\frac{\Phi_{\mathrm{B}, \text { crit }}}{\Phi_{\mathrm{B}}}\right)^{2} \quad \mathrm{yr},
\end{aligned}
$$

where $\Phi_{\mathrm{B}}$ is the actual flux of the core at any stage past the onset of dynamical contraction, and $\Phi_{\mathrm{B} \text { crit }}$ is uniquely determined by the mass of the core from equation (1) as $\Phi_{\mathrm{B}, \text { crit }}=$ $(63 G)^{1 / 2} M$. The normalization of $n_{\mathrm{i}} / n_{\mathrm{H} 2}$ in equation $(7 \mathrm{~b})$ refers to a neutral density $\sim 10^{9}$ $\mathrm{cm}^{-3}$, above which $n_{\mathrm{i}}$ no longer increases with $n_{\mathrm{n}}$ (see Elmegreen 1979; Nakano 1979).

It is emphasized that equation (7) refers to a core, not to the entire cloud. The cloud as a whole has $M \simeq M_{\text {crit }}$ because it is magnetically supported. However, a core of mass $\sim 1$ $M_{\odot}$ has $M<M_{\text {crit }}$ or, equivalently, $\Phi_{\mathrm{B}}>\Phi_{\mathrm{B}, \text { crit }}$ for a relatively long time, i.e., until the quasistatic phase of ambipolar diffusion manages to reduce the flux of this (fixed) mass so as to make the mass-to-flux ratio equal to its critical value. If this marks the onset of accelerated contraction of the core (see eq. [2b]), then equation (7a) shows clearly that this will also mark the stage at which $\tau_{\mathrm{AD}}$ begins to increase above its quasistatic contraction value by the factor $\left(\Phi_{B, c r i t} / \Phi_{B}\right)^{2}$. It therefore becomes more difficult to lose flux during this late phase of accelerated contraction. Although ambipolar diffusion may have reduced the flux of such a core by a few orders of magnitude prior to this stage, equation (1) yields a critical flux for a $3 \mathbf{M}_{\odot}$ core which is larger than the observed fluxes of magnetic stars by a factor 10 - 50. Hence, it seems that ambipolar diffusion may not resolve the entire magnetic flux problem of star formation. To complete the task, then, one may need to appeal to another dissipative process (such as ohmic dissipation) that sets in at higher densities, at which the electron density becomes negligible and the negative charge is mainly carried by grains (Spitzer 1963). Ohmic dissipation at densities $\approx 10^{12} \mathrm{~cm}^{-3}$ has recently been studied by Nakano and Umebayashi (1986), who conclude that it can reduce the protostellar flux to the desired level prior to flux re-freezing (originally studied by Pneuman and Mitchell 1965) due to thermal ionization. Detailed collapse calculations beyond the density $\sim 10^{9} \mathrm{~cm}^{-3}$, accounting for the significant reduction in the electron density and the fact that collisions no longer permit the dominant charge carriers to remain attached to the magnetic field, are essential for understanding precisely how nature resolves the magnetic flux problem.

Equation (7) taken at face value implies that, if quasistatic contraction progresses to a density $n_{\mathrm{n}} \sim 10^{9} \mathrm{~cm}^{-3}$ (not an unreasonable proposition given the conclusions by Mouschovias [1976b, 1978] concerning the effective support against gravity by magnetic tension, confirmed also by Nakano's 1979 quasistatic calculation), a core's flux may decrease by at least another order of magnitude prior to the stage $n_{\mathrm{n}} \sim 10^{12} \mathrm{~cm}^{-3}$. This is so because we now have that (i) $\tau_{\mathrm{AD}} / \tau_{\mathrm{ff}} \propto \rho_{\mathrm{n}}^{-1 / 2} \Phi_{\mathrm{B}}^{-2}$ (since $n_{\mathrm{i}} \simeq$ const above $n_{\mathrm{n}} \simeq 10^{9} \mathrm{~cm}^{-3}$ ), and (ii) the charged particles themselves are not well attached to the magnetic field. Hence, ambipolar diffusion may still have its day in resolving the magnetic flux problem.

Although subAlfvénic collapse of cloud cores is the most common case (see Mouschovias 1978, App. B), Alfvénic or superAlf vénic collapse (e.g., due to implosion) leads to a very different sequence of events and, most likely, to a different IMF; i.e., more high-mass stars.

\subsection{SubAlfvénic Collapse}

1.3.1. Redistribution of Angular Momentum and Magnetic Flux, and Core-Envelope Separation. The tension of the field lines effectively supports the outlying portions of a cloud, thus forming an extended envelope, while allowing a dense central core to develop and a highly nonhomologous contraction to set in once gravitational forces become strong 
enough (see Mouschovias 1976b). During the initial stages of contraction, magnetic braking is so effective that the cloud contracts at nearly constant angular velocity, comparable to that of galactic rotation. (Thus the kinetic energy of rotation decreases as $R^{2}$, and the centrifugal force decreases as $R$.) Since magnetic braking becomes somewhat less effective at densities $\sim 10^{4} \mathrm{~cm}^{-3}$ due to slow onset of ambipolar diffusion and the development of a larger density contrast between a core and the envelope (see Mouschovias 1978, § IId), two phenomena should reveal themselves at such densities. (i) A cloud core (or a molecular cloud with a dense envelope) should spin up noticeably above the rate of rotation that it would have if it remained in a synchronous galactocentric orbit at all stages of contraction --but still well below the rate of rotation implied by angular momentum conservation from an initial density $n \sim 1 \mathrm{~cm}^{-3}$ and angular velocity $\Omega \sim 10^{-15} \mathrm{rad} \mathrm{s} \mathrm{s}^{-1}$. [Observed angular velocities are at least one (and usually two or more) order(s) of magnitude smaller than those implied by conservation of angular momentum (see review by Goldsmith and Arquilla 1985, and references therein). There is little doubt that magnetic braking has been at work from scales larger than $\sim 10 \mathrm{pc}$ to scales smaller than $\sim 10^{-2} \mathrm{pc}$.] (ii) The central flux tubes of a cloud should keep increasing their mass-to-flux ratio due to ambipolar diffusion.

1.3.2. Fragmentation, Disk Formation, and Bipolar Outflows. Even with the magnetic field still frozen in the matter, fragments with $M_{\mathrm{fr}} \sim 150 \mathrm{M}_{\odot}$ can separate out in a cloud of mass $M \sim 10^{4} \mathrm{M}_{\odot}$ at a density $n\left(=n_{\mathrm{p}} / 2\right) \sim 10^{4} \mathrm{~cm}^{-3}$--recall that equation (1) yields

$$
M_{\text {crit }}=5.0 \times 10^{5} \frac{(B / 3 \mu \mathrm{G})^{3}}{\left(n_{\mathrm{p}} / 1 \mathrm{~cm}^{-3}\right)^{2}} \quad \mathrm{M}_{\odot},
$$

(see Mouschovias and Spitzer 1976; Spitzer 1978; Mouschovias 1978). As ambipolar diffusion increases the mass-to-flux ratio in a fragment (or core) above the critical value for collapse, lower-mass fragments separate out gravitationally and begin to contract on their own. For example, even with a magnetic field as large as $\sim 100 \mu$ Gauss in a core in which ambipolar diffusion is in progress, equation (8) implies that fragments with $M_{\mathrm{fr}} \leqslant M_{\text {crit }} \simeq 3 \mathrm{M}_{\odot}$ can separate out at neutral densities $n_{\mathrm{n}} \sim 4 \times 10^{4} \mathrm{~cm}^{-3}$. A plethora of low-mass, low-spin fragments (or cores) should therefore be forming preferentially in (and perhaps only in) the cores of self-gravitating clouds which (clouds) contract either subAlf vénically or not at all, while more massive fragments can form further out from a cloud's core (see also Mouschovias 1978 for the same, but somewhat less refined, prediction). This is consistent with observations of low-mass fragments in molecular clouds (see review by Myers 1985).

That a contracting core should form a disk with its axis of symmetry aligned with the common direction of the magnetic field and the angular momentum vectors is an inevitable consequence of the inherent anistropy of magnetic and centrifugal forces. However, there is as yet no theory that can predict, as opposed to assume, what the distribution of mass in the disk should be and what fraction of that mass should eventually find its way into a protostar. Neither is there as yet a theory that can predict, or just explain, the commonly observed bipolar outflows in regions of low-mass star formation.

\subsubsection{Formation of Binary Stars and of Intermediate- and Low-Mass Stars in Cloud} Cores, and Selection of $\sim 1 \mathrm{M}_{\odot}$ as a Typical Stellar Mass. For these subAlfvénically contracting cores, it was demonstrated early on that magnetic braking followed by ambipolar diffusion can explain the entire range of periods of binary stars from $10 \mathrm{hr}$ to $100 \mathrm{yr}$ (Mouschovias 1977). Moreover, a single maximum in the distribution of the number of binaries as a function of (orbital) period was predicted, in disagreement with earlier but in agreement with more recent observations (see review by Abt 1983). It has also been shown recently that, in subAlf vénically contracting cores, magnetic braking can be effective even 
while ambipolar diffusion is in progress, so that sufficient angular momentum is lost to the envelope for Sun-Jupiter "binaries" and single stars to form without interference from centrifugal forces (see Mouschovias and Paleologou 1986; or Mouschovias 1987b, § 4).

In the case of magnetically connected fragments, trapping of torsional Alfven waves sets each one of them in a series of high-spin and low-spin states (Mouschovias and Morton $1985 \mathrm{a}, \mathrm{b})$. Thus the angular momentum problem for each fragment may be resolved, recreated, and then resolved again, perhaps several times, before the angular momentum of the system of fragments is reduced sufficiently for stars to form.

Since low-mass fragments owe their existence to ambipolar diffusion, they reach the dynamical stage of contraction and protostar formation before the more massive, outlying fragments, which may have formed while the magnetic field is nearly frozen in the matter. However, once a massive star has formed, it evolves relatively rapidly and either the expansion of the resulting H II region or the eventual supernova explosion changes the evolution of other, especially massive, fragments substantially. In particular, a supernova may induce rapid collapse of nearby fragments with masses $\sim 10^{1}-10^{3} \mathrm{M}_{\odot}$, while it may disperse the least massive ones, and may lead to more efficient star formation.

The magnetic field unavoidably introduces the length scale $\lambda_{\mathrm{A}} \simeq \pi v_{\mathrm{A}} \tau_{\mathrm{ni}}$ in molecular clouds [see Mouschovias 1987a, § 2.2.6(b); Mouschovias, Morton, and Ciolek 1990] below which ambipolar diffusion prevents Alfvén waves from propagating in the neutrals. This, in combination with a thermal and a magnetic Jeans length $\left(\lambda_{\mathrm{T}}=1.1 C_{\mathrm{a}} \tau_{\mathrm{ff}}\right.$ and $\lambda_{\mathrm{M}}=0.6 v_{\mathrm{A}} \tau_{\mathrm{ff}}$, respectively, where $C_{\mathrm{a}}$ is the adiabatic speed of sound), selects $1-0.1 \mathrm{M}_{\odot}$ as natural masses for protostellar cores at the respective density range $10^{4}-10^{6} \mathrm{~cm}^{-3}$. For primordial chemical composition, the magnetically selected masses are $z 10 \mathrm{M}_{\odot}$, implying that massive stars may have been the first to form in protoglobular cluster clouds. Since these stars can reach the supernova stage reletively rapidly and eject heavy elements into the parent cloud before low-mass stars form, the observed metal contamination of low-mass stars in globular clusters (see review by Wheeler et al. 1989) may thus find a natural explanation (Mouschovias 1989).

\subsection{Alf vénic or SuperAlf vénic Collapse}

Both theoretically and observationally such rapid collapse of a cloud as a whole should be a very rare phenomenon. It should happen, if at all, mostly in relatively massive fragments imploded by a sudden and large increase in the external pressure. [The sequence of events described here or in Mouschovias 1987a, § 3.6, differs qualitatively, quantitatively, and in specificity from ideas concerning "bimodal" star formation under the assumption that rapid collapse and formation of massive stars are the result of $M>M_{\text {crit }}$ (e.g., contrast this discussion with that in Lizano and Shu 1987), in which case the magnetic tension force increases rapidly with contraction (Mouschovias 1978).] During the rapid implosion of a clump with mass $M \simeq M_{\text {crit }}$ in a cloud complex, magnetic tension cannot stop or significantly slow down the collapse and, in addition, the torsional Alfven waves remain trapped within the clump. Contraction with angular momentum nearly conserved implies that centrifugal forces become progressively more important. Depending, however, on the precise density at which rapid collapse begins, they may not increase sufficiently to prevent the formation of very wide binaries, including rapidly rotating, massive single stars as members. For example, if angular momentum begins to be conserved above a density $\simeq 10^{4} \mathrm{~cm}^{-3}$, the angular momentum left over in a fragment (due to earlier efficient magnetic braking) is exactly what is required to form a relatively wide (visual) binary system, with a period $\tau_{b} \simeq$ 100 yr. In fact, a simple model shows that binaries forming according to this scenario will have periods scaling with the density $n_{\mathrm{cr}}$, above which angular momentum is nearly conserved, as $\tau_{\mathrm{b}} /(100 \mathrm{yr}) \simeq\left[\left(10^{4} \mathrm{~cm}^{-3}\right) / n_{\mathrm{cr}}\right]^{2}$ (Mouschovias 1977). Note that conservation of angular momentum during superAlf vénic collapse is due to trapping of the torsional Alfven 
waves inside the cloud, not due to rapid ambipolar diffusion. This is a qualitative difference from our early work, and has significant implications for massive star formation.

If Alf vénic or superAlfvénic contraction begins at $n<10^{3} \mathrm{~cm}^{-3}$, centrifugal forces will lead to a quasi-equilibrium configuration by balancing gravity perpendicular to the axis of rotation. Further contraction takes place only as rapidly as magnetic braking can remove angular momentum to the envelope. Since the fragment still has most of its magnetic flux trapped inside at this stage and since it was magnetically supported before implosion, magnetic forces will also contribute significantly to this quasi-equilibrium, and both magnetic braking and ambipolar diffusion are expected to occur simultaneously, probably over comparable time scales. Stars which will form in such fragments will be characterized by both rapid rotation and strong magnetic fields. Since these are signatures of massive stars, it is tempting to suggest, at least tentatively, that this is how such stars form (see Mouschovias 1987a, 1989, for a strengthening of this suggestion and for a scenario of highmass star formation even in subAlfvénically contracting but very massive clouds).

\subsection{The $B_{c}-\rho_{c}$ Relation in the Presence of Ambipolar Diffusion}

Detailed calculations show that, after an initial flattening of a cloud along field lines (which does not appreciably increase the field strength), the field $B_{c}$ increases as $\rho_{c}{ }^{1 / 2}$ although ambipolar diffusion is in progress (see Fiedler and Mouschovias 1990). In other words, the relation obtained earlier for self-gravitating clouds with frozen-in fields, namely,

$$
B_{\mathrm{c}} / B_{0}=\left(\rho_{\mathrm{c}} / \rho_{0}\right)^{1 / 2}, \quad n_{0} \simeq 137\left(B_{0}{ }^{3} / M\right)^{1 / 2} \mathrm{~cm}^{-3} \text {, }
$$

(Mouschovias 1976b) can be used even in the presence of ambipolar diffusion at least up to densities $\sim 10^{9} \mathrm{~cm}^{-3}$ although the physics enforcing this relation is quite different in the two situations. The quantity $\rho_{0}$ in equation $(9 a)$ is the gas density at which gravitational forces become comparable to magnetic forces (at field strength $B_{0}$ ) and significant contraction perpendicular to field lines begins. Converted to a number density of protons $\left(n_{0}\right)$, it is approximately given by equation $(9 \mathrm{~b})$, where $B_{0}$ is measured in microgauss and the cloud's mass $M$ in solar masses (see brief review by Mouschovias 1985). Equation (9b) yields a density range $n_{0} \simeq 1-320 \mathrm{~cm}^{-3}$ for $B_{0}=3 \mu$ Gauss and for $M$ in the corresponding range $5 \times 10^{5}-5 \mathrm{M}_{\odot}$. The exponent $\kappa$ in the $B_{\mathrm{c}} \propto \rho_{\mathrm{c}}{ }^{\kappa}$ relation is essentially zero below the density $n_{0}$ and becomes equal to $1 / 2$ above $n_{0}$. (For the spatial variation of $\kappa$ within a cloud and its physical explanation, see Mouschovias 1978, § IIIa2.) We find that, typically, the central field is enchanced by a factor $10^{2}$, so that its value becomes $\simeq 3 \mathrm{mG}$ at a density $\simeq 3 \times 10^{8} \mathrm{~cm}^{-3}$. The region of strong field involves a contracting core mass $s 0.1 \mathrm{M}_{\odot}$. The strong fields measured in $\mathrm{H}_{2} \mathrm{O}$ masers are consistent with the predictions of our calculations.

\section{SUMMARY}

Cosmic magnetism plays a crucial role in star formation. Aside from its role in the formation of interstellar clouds, it is at first gravity's most faithful ally in reducing the otherwise potentially fierce centrifugal forces to near insignificant levels (through magnetic braking). Later, it becomes gravity's most formidable opponent and converts the would-be violent star formation process into a relatively quiescent one for most of its duration. Even as ambipolar diffusion, the means which gravity devises to win its battle against magnetic fields, allows the mass-to-flux ratio $\left(d m / d \Phi_{B}\right)_{c}$ of cloud cores to increase, magnetic forces still regulate the contraction rate even beyond the stage at which $\left(d m / d \Phi_{B}\right)_{c}$ exceeds the critical value $(63 G)^{-1 / 2}$. Calculations suggest a tentative theory of star formation which accounts naturally for the formation of close binaries, intermediate- and low-mass stars, and 
Sun-Jupiter "binaries" in subAlfvénically contracting clouds, for massive stars in superAlfvénically contracting clouds, and for wide binaries in both kinds of clouds.

Aligned rotators are more common in nature than perpendicular rotators because the relative magnitude of the magnetic braking time scales for dense clouds (or cores) is $\tau_{\|} / \tau_{\perp}=$ $0.5\left(\rho_{\mathrm{cl}} / \rho_{\text {ext }}\right)^{1 / 2}$. During quasistatic contraction of an axisymmetric cloud, the ambipolar diffusion time scale in the core is $\tau_{\mathrm{AD}}=0.6 \tau_{\mathrm{ff}}{ }^{2} / \tau_{\mathrm{ni}}$, where $\tau_{\mathrm{ff}}$ is the free fall time scale and $\tau_{\text {ni }}$ the mean collision time of a neutral particle with ions. After dynamical contraction begins in the core, we find that $\tau_{\mathrm{AD}}=0.3\left(\tau_{\mathrm{ff}}{ }^{2} / \tau_{\mathrm{ni}}\right)\left(\Phi_{\mathrm{B}, \mathrm{crit}} / \Phi_{\mathrm{B}}\right)^{2}$, where $\Phi_{\mathrm{B}, \text { crit }}=(63 G)^{1 / 2} M$ is the critical flux below which a core of mass $M$ may begin to contract dynamically. In typical cores of self-gravitating clouds, the magnetic field scales with the gas density as $B_{c} \propto \rho_{c}{ }^{1 / 2}$ even while ambipolar diffusion is in progress. Strong fields measured in $\mathrm{H}_{2} \mathrm{O}$ masers of mass s $0.1 \mathrm{M}_{\odot}$ and $n_{\mathrm{n}}>10^{8} \mathrm{~cm}^{-3}$ are consistent with recent collapse calculations.

Abt, H. A. 1983, Ann. Rev. Astr. Ap., 21, 343.

Blitz, L., and Shu, F. H. 1980, Ap. J., 238, 148.

Bregman, J. D., Troland, T. H., Forster, J. R., Schwarz, U. J., Goss, W. M., and Heiles, C. 1983, Astr. Ap., 118, 157.

Elmegreen, B. G. 1979, $A p$. J., 232, 729.

Fiedler, R. A., and Mouschovias, T. Ch. 1990, Ap. J., to be submitted.

Field, G. B. 1965, Ap. J., 142, 531.

Goldsmith, P. F., and Arquilla, R. 1985, in Protostars \& Planets II, eds. D. C. Black, and M. S. Matthews (Tucson: Univ. of Arizona Press), p. 137.

Heiles, C. 1987, in Physical Processes in Interstellar Clouds, eds. G. E. Morfill and M. Scholer (Dordrecht: Reidel), p. 429.

Jeans, J. H. 1928, Astronomy and Cosmogony (Cambridge: Cambridge Univ. Press).

Lizano, S., and Shu, F. H. 1987, in Physical Processes in Interstellar Clouds, eds. G. E. Morfill and M. Scholer (Dordrecht: Reidel), p. 173.

McDaniel, E. W., and Mason, E. A. 1973, in The Mobility and Diffusion of Ions in Gases (New York: Wiley).

Mestel, L., and Spitzer, L., Jr. 1956, M. N. R. A. S., 116, 503.

Mouschovias, T. Ch. 1974, Ap. J., 192, 37.

1975a, Astr. Ap., 40, 191.

1975b, Ph.D. Thesis, Univ. of California at Berkeley.

1976a, Ap. J., 206, 753.

1976b, Ap. J., 207, 141.

1977, Ap. J., $211,147$.

1978, in Protostars and Planets, ed. T. Gehrels (Tucson: U. of Ariz. Press), p. 209.

1979a, Ap. J., 228, 159.

1979b, Ap. J., 228, 475.

1981, in Fundamental Problems in the Theory of Stellar Evolution, eds. D. Sugimoto, D. Q. Lamb, and D. N. Schramm (Dordrecht: Reidel), p. 27.

1983, in Solar and Stellar Magnetic Fields: Origins and Coronal Effects, ed. J. O. Stenflo (Dordrecht: Reidel), p. 479.

1985, Astr. Ap., 142, 41.

1987a, in Physical Processes in Interstellar Clouds, eds. G. E. Morfill and M. Scholer (Dordrecht: Reidel), p. 453.

- 1987b, in Physical Processes in Interstellar Clouds, eds. G. E. Morfill and M. Scholer (Dordrecht: Reidel), p. 491.

1989, in The Physics and Chemistry of Interstellar Molecular Clouds, eds. G. Winnewisser and J. T. Armstrong (Berlin: Springer-Verlag), p. 297. 
Mouschovias, T. Ch., and Morton, S. A. 1985a, Ap. J., 298, 190.

. 1985b, Ap. J., 298, 205.

. 1990, Ap. J., to be submitted.

Mouschovias, T. Ch., Morton, S. A., and Ciolek, G. 1990, in preparation.

Mouschovias, T. Ch., and Paleologou, E. V. 1979, Ap. J., 230, 204.

. 1980, Ap. J., 237, 877.

. 1986, Ap. J., 308, 781.

Mouschovias, T. Ch., Paleologou, E. V., and Fiedler, R. A. 1985, Ap. J., 291, 772.

Mouschovias, T. Ch., and Spitzer, L., Jr. 1976, Ap. J., 210, 326.

Mouschovias, T. Ch., Shu, F. H., and Woodward, P. R. 1974, Astr. Ap., 33, 73.

Myers, P. C. 1985, in Protostars \& Planets II, eds. D. C. Black, and M. S. Matthews (Tucson: Univ. of Arizona Press), p. 81.

Nakano, T. 1979, Publ. Astr. Soc. Japan, 31, 697

Nakano, T., and Umebayashi, T. 1986, M. N. R. A. S., 218, 663.

Paleologou, E. V., and Mouschovias, T. Ch. 1983, Ap. J., 275, 838.

Parker, E. N. 1966, Ap. J., 145, 811.

Pneuman, G. W., and Mitchell, T. P. 1965, Icarus, 4, 494.

Schwarz, U. J., Troland, T. H., Albinson, J. S., Bregman, J. D., Goss, W. M., and Heiles, C. 1986, Ap. J., 301, 320.

Shu, F. H. 1974, Astr. Ap., 33, 55.

. 1983, Ap. J., 273, 202.

Shu, F. H., Adams, F. C., and Lizano, S. 1987, Ann. Rev. Astr. Ap., 25, 23.

Spitzer, L., Jr. 1962, Physics of Fully Ionized Gases, 2nd ed. (New York: Interscience). . 1963, in Origin of the Solar System, eds. R. Jastrow and A. G. W. Cameron (New York: Academic Press), p. 39.

- 1978, Physical Processes in the Interstellar Medium (New York: Wiley-Interscience).

Tomisaka, K., Ikeuchi, S., and Nakamura, T. 1988, Ap. J., 335, 239.

Wheeler, J. C., Sneden, C., and Truran, J. W., Jr. 1989, Ann. Rev. Astr. Ap., in press.

DUDOROV: How can you explain that low-mass stars are formed in dark clouds with temperature $\mathrm{T} \simeq 10 \mathrm{~K}$ and magnetic field $\mathrm{B}<\mathrm{a}$ few times $10 \mu \mathrm{G}$ and also in giant molecular clouds with $\mathrm{T} \simeq 20 \mathrm{~K}$ and $\mathrm{B} \simeq 100 \mu \mathrm{G}$, but massive stars are formed only in giant molecular clouds?

MOUSCHOVIAS: As you may have noticed in the scenario for star formation which I described, massive stars could form mainly through, at least initially, the super-Alfvenic collapse of fragments (see my diagram). This, as I explained, requires an implosion of a fragment (e.g. either by a supernova shock wave or by the pressurization of the region surrounding the fragment by an HII region). Causes for implosion are found in molecular clouds but not in dark clouds.

KAHN: It is important to know $G_{i}$ (rate of creation of ions) and $L_{i}$ (rate of loss of ions) which seem to enter the equations of Mouschovias's scheme in such an innocuous manner. But these are highly uncertain quantities, and they spread uncertainty throughout the set of solutions. 